\title{
Pour une relecture dynamique de Senghor
}

\section{Boniface Mongo Mboussa}

\section{OpenEdition}

Journals

Édition électronique

URL : http://journals.openedition.org/edl/2487

DOI : $10.4000 /$ edl.2487

ISSN : 2296-5084

\section{Éditeur}

Université de Lausanne

\section{Édition imprimée}

Date de publication : 15 décembre 2017

Pagination : 101-112

ISBN : 978-2-940331-66-6

ISSN : 0014-2026

\section{Référence électronique}

Boniface Mongo Mboussa, "Pour une relecture dynamique de Senghor », Études de lettres [En ligne], 3-4 | 2017, mis en ligne le 15 décembre 2019, consulté le 16 décembre 2020. URL : http:// journals.openedition.org/edl/2487 ; DOI : https://doi.org/10.4000/edl.2487 


\section{POUR UNE RELECTURE DYNAMIQUE DE SENGHOR}

La réflexion qui suit s'intéresse au cheminement de la pensée de Senghor au sortir de la Seconde Guerre mondiale. Loin de l'image du colonisé aliéné que certains intellectuels ont véhiculée durant plusieurs décennies, il s'agira de mettre en évidence à quel point les réflexions critiques et esthétiques de Senghor, trop souvent négligées, ont permis la progressive affirmation d'un réalisme africain, détaché du moule esthétique et politique qui imprégnait le discours français à ce moment-là. Il s'agit donc de clarifier un parcours qui s'est achoppé aux concepts antithétiques de la raison et de l'émotion, "gros mots» devenus signes d'une polarité ontologique indéfendable.

Lors d'un colloque qui s'est tenu le 26 juin 2006 à l'Assemblée nationale française sur l'héritage de Senghor, l'historien sénégalais Mamadou Diouf fait cet aveu édifiant:

Ma génération n’aimait pas beaucoup Senghor, parce qu'il était Président, parce qu'il parlait sans cesse de la France. [...]. De plus, on ne le lisait pas; on savait ce qu'il disait en lisant ce que d'autres écrivaient sur lui, les critiques les plus virulentes ${ }^{1}$.

Dans cette même veine d'une reconnaissance tardive, le philosophe béninois Paulin Houtondji, qui avait longtemps ignoré Senghor, reconnaît dans son autobiographie intellectuelle parue en 1997, Combats pour le sens, sa contribution à l'histoire de la pensée africaine et sa grande dignité $^{2}$; son compatriote Stanislas Adotevi, l'un des contempteurs les plus féroces du fondateur de la Négritude l'a réhabilité, à l'occasion des

I. J.-L. Debré, A. Diouf, Léopold Sédar Senghor, p. 204.

2. P. J. Houtondji, Combats pour le sens. 
quatre-vingt-dix ans du poète ${ }^{3}$. Récemment, l'écrivain nigérian Wole Soyinka, prix Nobel de littérature, auteur de la célèbre boutade contre la Négritude "le tigre ne revendique pas sa tigritude, mais il tue sa proie et la mange» ", a relu la poésie de Senghor à l'aune de l'actualité sudafricaine, celle du comité Vérité et Réconciliation, dont le pardon était le credo, et a décrété Senghor précurseur de cette démarche salvatrice. Senghor, écrit Wole Soyinka, "croyait que le pardon était essentiel dans une situation où la véritable justice ne peut être rendue ${ }^{5}$. Soyinka fait allusion ici au poème de Senghor "Prière de la paix" dans lequel il pardonne à l'Europe son inhumanité à l'égard de l'Afrique. Ce poème a suscité dans les années cinquante la désapprobation unanime des intellectuels africains, à cause de ces vers :

et je veux prier singulièrement pour la France.

Seigneur, parmi les nations blanches, place la France à la droite du Père ${ }^{6}$

Placés dans leur contexte d'énonciation, c'est-à-dire au cœur des luttes de décolonisation dans les années 50 , ces vers étaient considérés, au mieux comme une lâcheté - c'est en tous cas l'avis du philosophe camerounais Marcien Towa - au pire comme une trahison. Towa n'hésite pas à considérer que ce pardon procède d'un froid calcul:

D'aucuns seraient tentés de voir dans cette générosité extrême dans le pardon, une manière d'abandonner la lutte et les siens. D'autres y chercheraient plus volontiers une ruse subtile, une fine diplomatie s'efforçant de toucher, de séduire par la hauteur d'âme et ainsi de gagner la confiance afin d'obtenir des réformes substantielles, mais sans heurt. [...] Il n'est donc pas interdit de penser que le pardon du poète est calculé, avec arrière-pensées réformistes ${ }^{7}$.

Pourtant, l'origine de ce choix du pardon est bel et bien la Seconde Guerre mondiale. Le 20 juin 1940, soit deux jours avant l'armistice, Senghor, soldat du troisième régiment de l'infanterie coloniale, est fait prisonnier à La Charité-sur-Loire. Dès sa capture, les Allemands l'ont

3. S. Adotevi, "L'autre Senghor», p. 31.

4. Cité par L. Kesteloot, Anthologie négro-africaine, p. 259.

5. Cité par J. G. Vaillant, Vie de Léopold Sedar Senghor, p. 16.

6. L. S. Senghor, Euvre poétique, p. 99.

7. M. Towa, Négritude ou servitude, p. 21. 
sorti du rang, ainsi que d'autres tirailleurs sénégalais, puis aligné le long du mur. Courageusement, un officier français entre en scène et en appelle à l'honneur des Allemands. Senghor, sauvé, est déplacé de camp en camp et c'est dans l'un d'eux, à Poitiers, au Frontslag 230, qu'il écrit Hosties noires, carnet de prison contenant "Prière de la paix", le poème de la discorde. Tout en rédigeant, Senghor lit Platon, médite sur ce qu'il nomme le "miracle grec», un métissage culturel et biologique. De cette lecture et de sa captivité date son passage de la Négritude-ghetto à la Négritude comme enracinement et ouverture.

Notre réflexion s'attardera sur le cheminement de la pensée de Senghor à cette époque, car celui-ci implique autant une capacité à pardonner, à transmettre l'expérience par la poésie, qu'à discuter de la forme même de cette transmission. Il s'agit donc de mettre au jour ce que nombre d'intellectuels contemporains de Senghor ont omis en ne voyant en lui qu'une figure figée, celle d'une forme de compromission incompatible avec la révolte à mener. La lecture rétrospective proposée veut dépasser les clivages idéologiques de ce temps pour mettre en évidence les enjeux négligés des réflexions esthétiques du poète, trop longtemps réduites à l'opposition "ontologique» de la raison et de l'émotion. De fait, notre démarche permettra de reconnaître ce que l'engagement de Senghor a réalisé: la progressive affirmation d'un réalisme africain, détaché d'un moule esthétique marqué par le discours français politisé de l'après-Seconde Guerre mondiale.

\section{Hosties Noires, une pédagogie du pardon}

Revenant sur son expérience de guerre, Senghor précise:

Pendant la captivité, le triomphe du nazisme nous avait forcés à ouvrir les yeux. Nous avons commencé à nous apercevoir, la lecture et la réflexion aidant, que toutes les grandes civilisations, sans exception, avaient été des civilisations de métissage biologique et culturel. Je dois dire cependant que je n'ai jamais eu de haine contre les Allemands. Je me suis même mis à apprendre l'allemand, et au bout de deux ans, je parlais mieux l'allemand que l'anglais. [...] Ainsi ai-je toujours fait la distinction entre le peuple allemand et le nazisme. J'ai réuni les 
poèmes que j'avais écrits en captivité sous le titre d'Hosties Noires. Vous n'y lirez pas un seul mot de haine ${ }^{8}$.

Lu dans cette perspective, le vers tant vilipendé s'éclaire. Senghor n'a jamais caché combien durant toute sa vie il s'est évertué à concilier ses deux identités française et africaine. Mais ce qui nous interpelle ici est comment l'expérience de la guerre a façonné l'homme. La guerre, mère de toute chose disait Héraclite ${ }^{9}$, a été l'expérience humaine majeure de Senghor. Cela ne surprend guère. D’autres avant lui l'ont médité. Walter Benjamin - qui n'a jamais été soldat - a mieux que quiconque montré l'impact de la Première Guerre dans la vie de toute une génération "qui était allée à l'école en tramway à chevaux" ${ }^{10}$, et qui, au retour des champs de bataille, était frappée de mutisme, incapable de narrer son expérience, c'est-à-dire d'assumer la transmission indispensable à la pérennité des sociétés.

Si dans l'immédiat, Senghor réussit à écrire un recueil de poèmes - une sorte d'histoire immédiate - célébrant la mémoire de ses frères d'armes et donc à assumer une forme de transmission, il montrera plus tard, lors du débat opposant Césaire à Aragon sur la question de la poésie nationale, l'incapacité du réalisme à dire le monde après la tragédie de la Seconde Guerre mondiale. Rappelons les faits pour saisir l'enjeu de son propos. Nous sommes en 1953, Aragon, auréolé de son prestige de résistant publie trois poèmes composés conformément aux règles traditionnelles de la versification française. Ils sont accompagnés d'une présentation: «D'une poésie nationale, et de quelques exemples» dans laquelle il justifie pourquoi, depuis l'Occupation, certains poètes ont eu recours aux formes traditionnelles de la versification. L'enjeu de la démarche est précis puisqu'il s'agit de mettre en cause «l'individualisme formel en poésie" pour se rallier à une nécessité de défendre des "valeurs françaises", nationales et esthétiques. À la fin de l'année 1954, Aragon réunit en volume sous le titre Journal d'une poésie nationale l'ensemble de ses articles, suivi d'une anthologie de textes illustratifs. Ce débat lancé par Aragon serait, comme le dit Bernard Mouralis, resté circonscrit au cadre strict de l'histoire française si le poète haïtien René Depestre

8. L. S. Senghor, La poésie de l'action, p. 85 sq.

9. "Fragments d'Héraclite», cités par C. Magris, Trois contes, récits de voyages, p. 83.

Io. Cité par G. Agamben, Enfance et histoire, p. 22. 
n'avait pas jugé bon de donner son avis ${ }^{11}$. Séjournant à l'époque au Brésil, il n'avait pas lu les articles d'Aragon parus dans les Lettres françaises, mais quand il les découvre dans Journal d'une poésie nationale, il jubile et transmet son enthousiasme au poète Charles Dobzynski dans une lettre que ce dernier publie justement dans les Lettres françaises ${ }^{12}$.

\section{Aragon, Césaire, Depestre et Senghor}

Depestre écrit :

Je suis en train de résoudre, grâce à Aragon, le conflit où se débattait mon «individualisme formel» [...] Je me suis théoriquement rallié aux enseignements décisifs d'Aragon et d'ici peu l'accord se fera entre la nouvelle conscience que j’ai acquise du réalisme en poésie et les moyens émotionnels par lesquels ma sensibilité est appelée à illustrer ma compréhension des problèmes soulevés et résolus dans le journal d'une poésie nationale ${ }^{13}$.

Celui-ci est donc convaincu qu'il faut associer création poétique et engagement politique en passant par un usage de formes soi-disant propres à une culture, une civilisation, une nation. Interpellé par la réaction de Depestre, Aimé Césaire l'apostrophe avec le poème «Réponse à Depestre, poète haïtien. Éléments d'un art poétique» et l'y somme de pratiquer, à l'instar de leurs ancêtres fugitifs, le marronnage, mode d'insoumission, avant de conclure:

Que le poème tourne bien ou mal sur l'huile de ses gonds

Fous-t-en Depestre fout-t-en laisse dire Aragon ${ }^{14}$

Paru dans les numéros 1-2 de la revue Présence Africaine (avril-juillet) 1955, alors que les Indépendances africaines ne sont encore qu'un fantasme, ce poème donne à la revue l'occasion d'initier un débat sur la «poésie nationale» auquel participe Senghor. À l'occasion de ces prises de position, chacun se justifie selon son expérience, sa culture et son

II. Voir B. Mouralis, Littérature et développement, p. 435.

I2. Ibid., p. 435.

I3. Ibid., p. 435.

I4. Ibid., p. 485. 
histoire. René Depestre s'appuie sur l'histoire d'Haïti à la fois française et caribéenne en affirmant une perspective communiste sur l'art, traduite par la mise au ban de l'«individualisme formel». Sur ce plan, il partage le même héritage que Césaire, mais celui-ci le reprend ironiquement en exploitant le registre propre aux communistes du moment:

Camarade Depestre

C'est un problème assurément très grave

des rapports de la Poésie à la Révolution

le fond conditionne la forme

et si l'on s'avisait aussi du détour dialectique

par quoi la forme prenant sa revanche

comme un figuier maudit étouffe le poème

mais non

Je ne me charge pas du rapport ${ }^{15}$

Léopold Sedar Senghor, lui, renvoie les communistes à leur idéologie littéraire: le réalisme socialiste. Le débat initié par Aragon sur la poésie nationale n'est en réalité que le prolongement de celui engagé en Union soviétique par Fadéev sur le réalisme socialiste. Senghor écrit:

On ne demande pas aux poètes de continuer le Parnasse ni aux peintres Corot et Courbet. Comme si les dictatures de l'ère atomique n'avaient pas mené l'humanité au bord du néant, comme si la relativité et la mécanique ondulatoire n’avaient pas changé, pour nous, plus que la face, l'essence même des choses. Et c'est l'an d'angoisse 1954, c'est l'an de grâce 1955 que choisissent nos théoriciens pour nous prêcher le retour qui au sonnet, qui au réalisme. S'ils s'adressaient encore à des Aryens... Que l'épithète de «socialiste» accompagne le substantif ne change rien aux données du problème ${ }^{16}$.

Non seulement il dénie au réalisme la capacité à dire le monde - et ce malgré sa prétention à l'objectivité, via le naturalisme -, mais doute même de son utilité comme genre littéraire et artistique, après 1945. Durant la guerre, Senghor n’a pas été un intellectuel passif, scrutant

I5. Ibid., p. 438.

16. Léopold Sedar Senghor et la revue Présence Africaine, p. 204. Le volume regroupe l'ensemble des articles de Senghor parus dans cette revue. L'article originel était paru dans le numéro de décembre 1955-janvier 1956. 
l'histoire en train de se faire; il a été un acteur, un témoin de ce que l'homme est capable de faire à l'homme. Et sur un mode mineur, il se demande comment écrire après la Seconde Guerre mondiale. Comment écrire à l'ère de la bombe atomique? Une question que devait poser de manière plus radicale Adorno, pour qui écrire un poème après Auschwitz relevait d'un acte barbare ${ }^{17}$.

Bernard Mouralis, puis Romuald Fonkoua ${ }^{18}$, ont rendu compte de ce débat initié par Aragon autour de la poésie nationale, impliquant évidemment la question des appartenances et l'affiliation à une origine culturelle et historique prédéfinie; ils ont mis en exergue les réactions de Césaire et Depestre, tout en excluant la contribution de Senghor, pourtant capitale. Capitale par sa lucidité, puisqu'elle convoque un autre débat, en 1955, entre le romancier camerounais Mongo Beti et l'écrivain guinéen Camara Laye touchant à la conception de la littérature engagée en Afrique. Mongo Beti reprochait à Camara Laye de produire une «littérature rose dans une Afrique noire» ${ }^{19}$ parce qu'il osait publier un roman d'apprentissage, L'enfant noir, qui ne dénonçait pas la colonisation. Face à cette critique, Senghor a répondu en affirmant que «l'art n'a pas de parti ${ }^{20}$. Cette réponse est capitale par sa dimension ontologique, car elle ne se contente pas de dénoncer l'inanité du réalisme à l'ère de la bombe atomique, mais propose aussi aux «écrivains nègres" de valoriser un nouveau réalisme, un réalisme africain cher à son compatriote Birago Diop qui, dans Les nouveaux contes d'Ahmadou Koumba, installe un univers où le fantastique et l'animisme côtoient sur un même plan le quotidien. Ce plaidoyer pour le réalisme africain n’a visiblement pas suffi à susciter l'adhésion, puisqu'aujourd'hui encore, Senghor est lu avec des a priori à cause du poème "Prière de Paix", mais aussi à cause de sa célèbre et malheureuse phrase «l'émotion est nègre comme la raison est hellène ${ }^{21}$ dont il importe de rediscuter.

I7. Th. Adorno, Prismes, p. 16.

I8. B. Mouralis, Littérature et développement. R. Fonkoua, Aimé Césaire, p. 164-183.

19. M. Beti, "Afrique noire littérature rose».

20. L. S. Senghor, «Liberté 1 », p. 159.

2I. Ibid., p. 24. 


\section{Ce que l'homme noir apporte: la sculpture nègre}

Tout commence par une sollicitation de l'écrivain catholique Daniel Rops qui demande à Senghor, pour la collection "Présences" aux éditions Plon, un essai sur «L'homme de couleur». Il s'agit pour Senghor, dans cet ouvrage collectif, d'évoquer la contribution de l'Afrique à la Civilisation. Senghor propose alors un essai intitulé à juste titre: "Ce que l'homme noir apporte». Grisé par ses lectures fécondes de l'époque (Frobenius, Hardy, Delafosse) célébrant l'Afrique, il ose le fameux alexandrin: «l'émotion est nègre comme la raison est hellène», puis passera sa vie à rattraper cette formule malheureuse. Tous ceux qui l'ont condamnée, parfois sans l'avoir lue, n’ont jamais cherché à la replacer dans son contexte. Nous sommes en 1939. Et la raison, depuis ce que Senghor appelle "La Révolution de 1889 ", c'est-à-dire depuis la publication de la thèse d'Henri Bergson ${ }^{22}$, depuis le mouvement Dada, depuis la psychanalyse qui découvre l'inconscient, depuis le surréalisme prônant l'amour fou et l'écriture automatique, est fortement malmenée en Europe. En amont, il y a Arthur Rimbaud qui s'est fait nègre dans Une saison en Enfer, sans oublier l'avènement de ce que Michel Leiris appelle «La crise nègre» ${ }^{23}$ dans le monde occidental, soit la vogue de l'art nègre en Occident. Enivré par toutes ces remises en cause de la raison, et donc de l'Occident, Senghor donne à lire son fameux alexandrin. Tous ceux qui critiquent son essai n'ont pas prêté attention aux deux exergues qui l'introduisent, l'un de Gide et l'autre de Paul Morand, célébrant tous deux la primauté de l'émotion sur la raison, ce qui conforte Senghor. Mais comme avec "Prière de paix», sa bonne foi n’a pas suffi à convaincre ses détracteurs. Voilà pourquoi, après la guerre, soit dix-sept ans plus tard, il tente de préciser sa pensée en la reformulant autrement. Dorénavant, il oppose «la raison intuitive» à «la raison raisonnante».

Dans un article sur l'esthétique négro-africaine paru dans la revue Diogène en 1956, il écrit en effet que "la raison européenne est analytique par utilisation, la raison nègre intuitive par participation " ${ }^{24}$. Plus tard encore, lors d'un colloque sur la Négritude à Dakar, il surenchérit:

22. H. Bergson, Essai sur les données immédiates de la conscience.

23. M. Leiris cité par J. Jamin, P. Williams, Une anthropologie du Jazz, p. 295.

24. Voir X. Garnier, "La notion de raison intuitive chez Léopold Sedar Senghor", p. 115 . 
Je le sais, les adversaires de la Négritude qui se prétendent marxistes me reprochent d'avoir écrit: «L'émotion est nègre comme la raison est hellène». Et d'en conclure que je dénie toute puissance de raisonnement, toute raison aux Nègres. Mais, comment savoir ce que j’ai voulu dire en isolant une phrase de son contexte? Car c'est l'évidence qu'ici "émotion" signifie raison intuitive, comme le mot soul chez les Negro Américains, et «raison» la raison européenne ${ }^{25}$.

Cette mise au point reste vaine. Finalement, c'est son compatriote le philosophe Souleymane Bachir Diagne qui met (provisoirement) fin à la polémique lorsqu'il publie en 2007 son essai Léopold Sedar Senghor, l'art africain comme philosophie. Il y propose une nouvelle approche de l'œuvre théorique de Senghor, en retrouvant la posture herméneutique adoptée par celui-ci "pour répondre à la question qui fut aussi celle de Picasso: que veulent dire les masques africains? Que disent ces objets que l'on a appelés des fétiches lorsque les dieux en sont partis? " ${ }^{26}$. C'est en partant de ces questions, nous apprend Souleymane Bachir Diagne, que Senghor a montré que les arts africains sont un langage et a mis au jour une ontologie dans laquelle l'être est rythme. Alors que Xavier Garnier dans son article "La notion de raison intuitive chez Senghor» situe l'origine de celle-ci chez l'ethnologue allemand Leo Frobenius ${ }^{27}$, et montre comment au fil des années Senghor réécrit et corrige sa formulation, Souleymane Bachir Diagne restitue le contexte dans lequel il avait formulé le célèbre alexandrin et oriente le débat sur l'art, plus précisément sur la sculpture africaine:

Quel est donc le contexte? Il écrit cette formule pour la première fois dans "Ce que l'homme noir apporte" en 1939, où il dresse la liste des contributions des Africains à la civilisation mondiale et où il analyse en particulier l'art africain. Il y oppose la sculpture africaine à la statuaire grecque. Il venait de lire le livre de Paul Guillaume et Thomas Munro ${ }^{28}$. Dans l'inspiration de ce qu'il avait lu, il établit une opposition frontale entre la statuaire grecque, qui, pour lui, repose sur l'imitation et une vision de l'humanité portée à sa perfection, à la sculpture africaine qui ne vise pas au plaisir

25. Ibid.

26. S. B. Diagne, Léopold Sedar Senghor, l'art africain comme philosophie, quatrième de couverture.

27. L. Frobenius, Histoire de la civilisation africaine.

28. P. Guillaume, Th. Munro, La sculpture nègre primitive. 
esthétique. L'émotion que l'on ressent devant une statuette est de nature différente. Voilà le contexte dans lequel il écrit cette formule. On comprend donc qu'il veut dire que l'émotion est à la sculpture africaine ce que la raison est à la statuaire grecque. La formule devient sous cet éclairage beaucoup moins scandaleuse ${ }^{29}$.

\section{Senghor aujourd'hui}

La réception de l'œuvre de Senghor par les intellectuels africains a donné lieu à plusieurs malentendus et élisions. Au cœur de la lutte pour les Indépendances, c'est-à-dire au mitan des années cinquante, son œuvre a été réduite au poème «Prière de paix» et à la formule "L'émotion est nègre comme la raison est hellène»; lors des Indépendances, les intellectuels ont souvent opposé sa négritude passive, "essentialiste», à celle de Césaire perçue comme davantage dynamique; quand il quitte le pouvoir en 1980 et devient académicien, Guy Ossito Midiohouan écrit un article assassin dans la revue Peuples Noirs Peuples Africains intitulé «La Gloire du collabo " ${ }^{30}$. À sa mort, Senghor ne fut célébré que comme fondateur de la francophonie, alors que l'ensemble de sa réflexion, remarquablement retracée dans Ce que je crois ${ }^{31}$, était négligée. L’heure semble venue de revisiter son œuvre avec davantage d'objectivité et de distance pour pouvoir reconnaître, comme l'avait fait son ami Aimé Césaire, que «Senghor est le lieu de rencontre de l'humanisme gréco-latin et de l'humanisme négro-africain. Il a transcendé l'antinomie apparente entre deux univers culturels différents et en a fait la synthèse vivante ${ }^{32}$.

Boniface Mongo Mboussa Sarah Lawrence College, Paris

29. S. B. Diagne, «Lire Marx avec les yeux de Teilhard», p. 86.

30. G. O. Midiohouan, «La gloire du collabo».

3I. L. S. Senghor, Ce que je crois, Négritude, Francité et civilisation de l'universelle.

32. A. Césaire, «Entretiens avec Le Monde», p. 200. 


\section{BIBLIOGRAPHIE}

Léopold Sedar Senghor et la revue Présence Africaine, Paris, Présence Africaine, 1996.

Adorno, Théodore, Prismes, traduit de l'allemand par Geneviève et Rainer Rochiltz, Paris, Payot, 1986.

Adotevi, Stanislas, "L'autre Senghor", in Présence de Senghor, éd. par Édouard Maunick, Paris, Éditions de l'UNESCO, 1997, p. 31-38.

Agamben, Giorgio, Enfance et histoire, traduit de l'italien par Y. Persant, Paris, Petite Bibliothèque Payot, 2002.

Bergson, Henri, Essai sur les données immédiates de la conscience, Paris, Felix Alcan, 1889.

Betr, Mongo, "Afrique noire, littérature rose», Présence africaine, 1-2 (avril-juillet 1955), p. 133-140.

CÉsaire, Aimé, «Entretiens avec Le Monde. 4. Civilisations », Paris, Le Monde/La Découverte, 1984, p. 193-201.

Debré, Jean-Louis, Diouf, Abdou, Léopold Sédar Senghor: la pensée et l'action politique, Actes du colloque organisé par la section française de l'Assemblée parlementaire de la Francophonie, Paris, 2006, en ligne: <http://www2.assemblee-nationale.fr/content/ download/16370/172299/version/1/file/colloque_senghor.pdf>.

Diagne, Souleymane Bachir, "Lire Marx avec les yeux de Teilhard", in Léopold Sédar Senghor: la pensée et l'action politique, éd. par JeanLouis Debré, Abdou Diouf, Actes du colloque organisé par la section française de l'Assemblée parlementaire de la Francophonie, Paris, 2006, p. 17-26 et 83-91 (discussions) et en ligne: <http:// www2.assemblee-nationale.fr/content/download/16370/172299/ version/1/file/colloque_senghor.pdf>.

-, Léopold Sedar Senghor. L'art africain comme philosophie, Paris, Riveneuve éditions, 2007.

Fonkoua, Romuald, Aimé Césaire, Paris, Seuil-Points, 2013 (2010).

Frobenius, Leo, Histoire de la civilisation africaine, traduit de l'allemand par Dr H. Back et D. Ermont, Gallimard, 1987 (1936). 
Garnier, Xavier, "La notion de raison intuitive chez Léopold Sedar Senghor", in Leopold Sedar Senghor. Africanité-Universalité, éd. par Jacques Girault et Bernard Lecherbonnier, Paris, L'Harmattan, 2002, p. 115-120 (Itinéraires et Contacts de cultures 31).

Guillaume, Paul, Munro, Thomas, La sculpture nègre primitive, Paris, Crès, 1929.

Houtondji, Paulin J., Combats pour le sens, Cotonou, Les Flamboyants, 1997.

Jamin, Jean, Williams, Patrick, Une anthropologie du Jazz, Paris, CNRS édition, 2016.

Kesteloot, Lilyan, Anthologie négro-africaine. Panorama critique des prosateurs, poètes et dramaturges noirs du XX $X^{e}$ siècle, Verdier, Marabout Université, 1967.

Magris, Claude, Trois contes, récits de voyages, traduit par J. et M.-N. Pastureau, Paris, Payot \& Rivages, 2006 (éd. originale L'infinito viaggiare, 2005).

Midiohounan, Guy Ossito, "La gloire du collabo", in Peuples Noirs Peuples Africains, 35 (Septembre-Octobre 1983), p. 72-73.

Mouralis, Bernard, Littérature et développement, Paris, Silex, 1984.

Senghor, Léopold Sedar, Négritude et humanisme, "Liberté 1 ", Paris, Seuil, 1964.

—, La poésie de l'action, Paris, Stock, 1980.

-, Ce que je crois, Négritude, Francité et Civilisation de l'universelle, Paris, Grasset, 1988.

—, Euvre poétique, Paris, Seuil, 1990.

Towa, Marcien, Négritude ou servitude, Yaounde, Clé, 1971.

Vaillant, Janet G., Vie de Léopold Sedar Senghor. Noir, Français et Africain, traduit de l'américain par R. Meunier, Paris, KarthalaSephis, 2006. 